\title{
Meteors and the Constitution of the Upper Air.
}

\section{By Prof. F. A. Lindemann, F.R.S.}

FROM the earliest times meteors and meteorites have probably been objects of wonder and interest. Astronomers have examined their radiants and established their connexion with certain comets, and physicists have been led by the study of their characteristics to quite unexpected conclusions about the conditions of the atmosphere at great heights. It is these latter developments that it is proposed to describe as briefly as possible.

Practically all meteorites which have been analysed are mixtures of metallic iron-nickel and of an olivinelike glass consisting of a double silicate of magnesium and aluminium, the proportions ranging from pure metal to pure glass. Since there is no real line to be drawn between meteorites and meteors, it seems legitimate to infer that meteors are composed of the same materials. Meteors appear at heights between I50 and 80 kilometres, and disappear at any height above ground-level, the meteorites of course actually reaching the ground. Their brightness varies within wide limits from the faint telescopic meteors invisible to the naked eye to the fire-balls said to rival the sun in brilliance, and their visible life may be anything between a fraction of a second and some fifteen seconds. Their recorded velocities range from some Io $\mathrm{km}$. $/ \mathrm{sec}$. to about roo $\mathrm{km}$. $/ \mathrm{sec}$., but it is clear from the nature of the case that these figures, especially in the higher ranges, cannot pretend to any great accuracy any more than can the estimates of luminosity. Deceleration along the path has never been certainly observed.

Presumably everybody would agree that meteors are extra-terrestrial particles moving with planetary velocities brought to incandescence by friction against the air. If further information, however, is desired, it is necessary to formulate somewhat more precisely what occurs when the meteor enters the atmosphere. To fix one's ideas it is as well to have in mind a typical meteor, for example, a meteor which appears at a height of roo kilometres and disappears at a height of 80 kilometres after traversing a path of 60 kilometres in $\mathrm{I} .5$ seconds. It may be assumed that this meteor appears as bright at a distance of 150 kilometres as a first magnitude star, i.e. that it radiates in the $I \cdot 5$ seconds of its visible life $3.3 \times 10^{10} \mathrm{ergs}$.

The first question to be examined is the original size of the meteor. It is easy to show that practically all its initial energy will be converted into radiation; for its speed relative to the air is so great, and collisions between particles moving at this speed and air molecules consequently so violent, that they disturb the electrons; sooner or later these must return to their normal orbits, entailing, of course, the emission of radiation. If all the energy appears as radiation it is simple to calculate the mass of the particle. The total energy is $3.3 \times 10^{10} \mathrm{ergs}$, the velocities $40 \mathrm{~km}$. $/ \mathrm{sec}$., so that its initial mass must have been $6 \cdot 25$ milligrams. If composed of iron, therefore, such a typical meteor would consist of a particle of diameter I. I 5 millimetres, i.e. about as large as a small shot.

It is now possible to examine what occurs when such a particle approaches the earth's atmosphere. At first the atmospheric molecules are so rare that the effect of one collision with a molecule will have been dissipated before the next occurs. The result of such collisions will be inappreciable. Most of the energy will be used up in ionising and breaking up the molecules, a part will be radiated, a smaller part will perhaps cause local heating and evaporation, but enough light to render the meteor visible cannot be produced. As the meteor approaches the earth, the density of the air increases and the frequency of these impacts becomes greater, until at a certain height the original colliding molecules and the evaporated meteoric molecules have not time to escape laterally from in front of the advancing meteor before they again collide with air molecules. It is in this region that a cap of gas begins to form in front of the particle which protects it from direct impacts and from loss of heat. Heat now begins to flow from this compressed and heated cap of gas into the particle, ultimately causing it to volatilise.

It is only when evaporation is appreciable that a meteor becomes visible. However hot it might be, a particle the size of which is measured in millimetres could not be seen at a distance of a hundred kilometres; when it evaporates its vapour spreads out and is brought to rest by collision with the molecules of the air in a comparatively large cross-section. The radiation emitted in this process from an effective surface measured in square centimetres is what is observed.

It may readily be seen how this qualitative description may be put upon a quantitative basis, and used to obtain information about conditions in the upper air. Meteors cannot appear until the cap of gas begins to form, i.e. until the chance of a molecule escaping laterally without further collision with an air molecule is small. We know the speed at which the meteor is travelling; we can make a fairly close estimate of the average lateral component of velocity of the escaping molecules; and, as shown above, we can calculate the size of any particular meteor the distance, brightness, duration and speed of which are known. Therefore we can calculate what the density of the air must have been if a cap was to be formed. This should represent a minimum value for the density at the height of appearance of the meteor. Fig. I shows the calculated densities plotted on a logarithmic scale against the height of appearance of sixty-five meteors the speed, brightness, etc., of which had been observed.

A second check may be attained by considering what happens after the cap has formed. A simple application of the kinetic theory of gases enables one to calculate what fraction of the total heat produced by the impact of the moving meteor upon the air will actually flow through the cap into the meteor and become available for heating and finally volatilising it. The total heat is of course equal to the amount of air accelerated in unit time, i.e. the product of the atmospheric density with the cross-section and velocity of the meteor, multiplied by one-half the square of its velocity. Hence the flow of energy available for heating or volatilising in terms of the atmospheric density can be determined. Since we are fairly certain of the size and constitution and consequently heat capacity and latent heat of each meteor, we can calculate the 
amount of heat necessary to raise it to a temperature at which evaporation takes place (the point at which the meteor should appear), and also the amount of heat necessary to complete the meteor's evaporation (the point at which the meteor should disappear). Therefore the heights of appearance and disappearance can

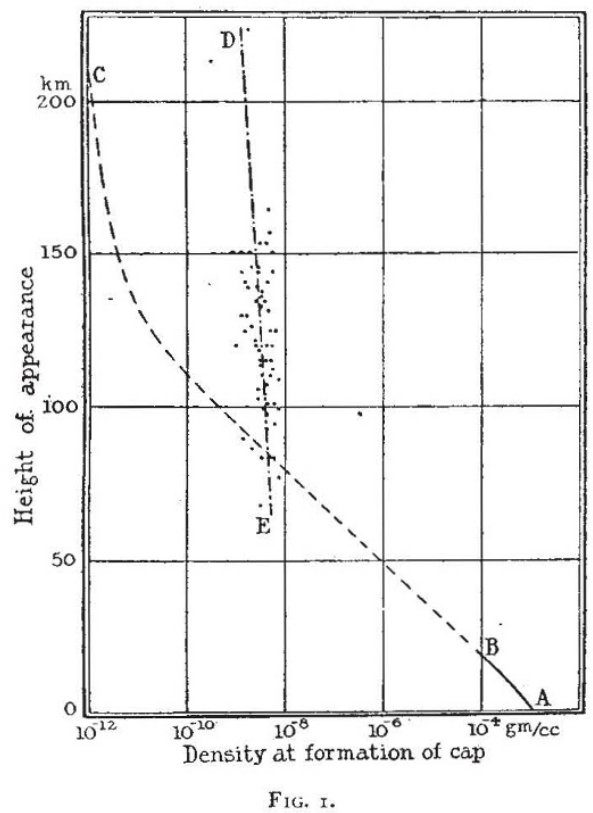

be calculated in terms of the observed meteoric characteristics and the atmospheric density, or inversely the atmospheric density can be calculated from the meteoric characteristics. Fig. 2 shows densities calculated on this basis for some hundred meteors plotted as crosses and dots against their heights of appearance and disappearance.

Though one could wish these points lay more closely upon a curve, their agreement may be regarded as satisfactory considering the uncertainty in the observational data, introduced more especially by the determination of the meteor's velocities. The two methods agree quite well among themselves, as is shown by the line DE repeated in Fig. 2 from Fig. $\mathrm{I}$.

In both figures the line $A B$ represents the density of the air derived from ballon sondes observation and the dotted line $\mathrm{BC}$ the density calculated, assuming the observed temperature of $220^{\circ} \mathrm{Abs}$. at a height of II kilometres to remain constant up to the confines of the atmosphere. It is clear that at great heights both methods give very much higher densities than correspond to this assumption. The only reasonable interpretation of the discrepancy seems to be that one is not justified in assuming that the temperature is constant from $25 \mathrm{~km}$. to $150 \mathrm{~km}$. simply because it has been shown to be constant between II $\mathrm{km}$. and $25 \mathrm{~km}$. In the accompanying figures the immediate observation of the temperature extends from $A$ to $B$. In view of the evidence obtained from meteors, it seems quite unjustifiable to extrapolate from $\mathrm{B}$ to $\mathrm{C}$. The meteor results would be satisfied if one assumed that the atmospheric temperature increased at heights above about 50 kilometres to something of the same order as the earth's surface temperature or perhaps somewhat higher.

This assumption may be supported by two lines of argument: the first is that further experimental facts seem to accord with it; the second is that it is to be expected a priori on the ground of known physical laws.

We may first consider four empirical facts which seem to agree with the view that the temperature at great heights is considerably higher than $220^{\circ}$ Abs., the first two based on observations of meteors, the last two upon quite independent phenomena.

As has been pointed out, meteorites consist almost without exception of two components, nickel-iron and a glassy olivine-like substance mixed in varying proportions. It has been shown that a meteor can only become visible if it evaporates; therefore the temperature in the cap of compressed gas in front of the meteor must attain a temperature at which iron or olivine evaporate if the meteor is to appear. Now the maximum temperature which gas can attain under adiabatic compression is readily calculated if one knows the initial temperature and the compression ratio. In the case we are considering it may be shown that the compression ratio is $3 v^{2} / 2 V_{0}^{2}, v$ being the meteor's velocity and $\mathrm{V}_{0}$ the molecular velocity. Hence the maximum temperature at the meteor's surface can be determined in terms of the velocity of the meteor and of the initial temperature of the air. Iron and olivine will only evaporate sufficiently rapidly to give rise to a visible meteor if the temperature is above $2000^{\circ}$. Meteors have been observed moving at a velocity of $12 \mathrm{~km}$. per sec. Our formula tells us that air compressed by a particle moving at $\mathbf{I} 2 \mathrm{~km}$. per sec. can only reach

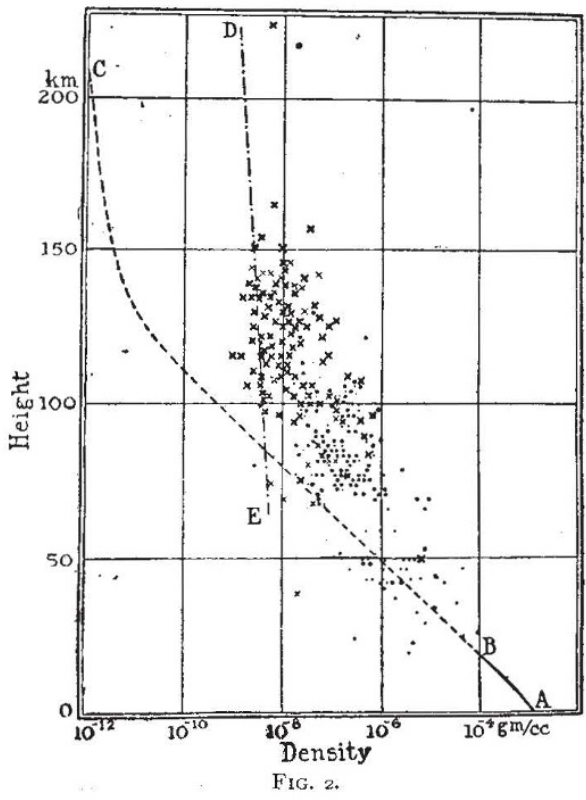

$2000^{\circ}$ if its initial temperature was $300^{\circ}$. This provides a very satisfactory and quite independent confirmation of the previous result.

A second somewhat more doubtful check may be found if the number of meteors which disappear at various heights is examined. It is found that whilst many meteors disappear between $100 \mathrm{~km}$. and $60 \mathrm{~km}$.

NO. 2962, VOL. II 8$]$ 
and many below $50 \mathrm{~km}$., scarcely any disappear between $50 \mathrm{~km}$. and $60 \mathrm{~km}$. So far only one reason has been put forward to account for this quite unexpected fact. As we have seen, the hotter the air is, the greater the flow of heat into the meteor. For a meteor approaching the earth there should be, on our theory, a region in which the air's initial temperature falls from $300^{\circ}$ to $220^{\circ}$, giving rise to a corresponding fall in the flow of heat. In this region one would expect a slower rate of evaporation and therefore diminished luminosity ; this effect would, however, soon be outweighed by the increased friction and heat production due to increased air density. It is clear that such a region is the most unlikely one for a meteor to disappear; if it reaches it at all it will probably penetrate into the cooler but denser atmosphere below. The only explanation for the fact that no meteors have been observed to disappear at $55 \mathrm{~km}$. seems to be that somewhere between say $52 \mathrm{~km}$. and $57 \mathrm{~km}$. the temperature of the air rises from the $220^{\circ}$ of the stratosphere to the higher temperatures in the neighbourhood of $300^{\circ}$ of the outer regions.

This view is confirmed in two very striking ways. As is well known, sources of sound are surrounded by alternate zones of audibility and silence. The distribution of these zones. has been explained by the assumption that the velocity of sound increases at great heights. Recent investigations have shown that the height at which this increase should occur in order to account for the observed facts is of the order of $50 \mathrm{~km}$. Clearly the well-known increase in velocity of sound with temperature would explain this otherwise inexplicable acoustical phenomenon if the view put forward above is correct, namely, that the temperature increases rapidly with height in this region.

A fourth argument in favour of this, though as yet not really a convincing one, is found in the well-known anomalies in the propagation of wireless waves. Notoriously the observed facts seem explicable only on the assumption that there is a conducting layer, the Heaviside layer, at a height varying from $50 \mathrm{~km}$. at sunset to some $80 \mathrm{~km}$. at sunrise. One can, with some plausibility, attribute this conducting layer to solar ionisation by day, but it seems difficult to account for its persistence at night otherwise than by assuming that the solar radiation forms some unstable compound which gradually reacts or breaks up forming ions during the hours of darkness. Such a substance is ozone, and it is clear, owing to the enormous increase in the rate of reaction with temperature, that the layer of maximum ionisation after sunset will be the lowest layer at which a high temperature exists, and that this will move up as the ozone is used up during the night to regions of lower density in which the rate of reaction is smaller. This hypothesis, of course, awaits many further checks, e.g. by observations at high latitudes during the polar night; but whatever the outcome, the observed fact that the height of the Heaviside layer after sunset lies between $50 \mathrm{~km}$. and $60 \mathrm{~km}$. seems very significant.

Finally, it might be worth while to discuss briefly the theoretical aspect of the high temperatures which observation seems to imply. Roughly speaking, above the troposphere, the region of convective equilibrium, the temperature is determined by radiation alone. Clearly a black or grey body would reach the mean temperature of the earth's surface, for this is the tem- perature at which emission balances absorption. The gases of the atmosphere are scarcely to be compared to black or grey bodies, however. The main constituents are, from the radiative point of view, somewhat dull and inert. Apart from the rather weak bands of oxygen in the red, neither oxygen, nitrogen nor argon has any well-marked absorption, and therefore emission bands at wave-lengths greater than $2000 \AA$.U., i.e. those to which practically all the sun's emission is confined. Infra-red absorption seems to be the function of the water-vapour, carbonic acid and the ozone. Now if one has a gas with strong absorption bands, even if mixed only in a small proportion with a neutral gas such as nitrogen, it will impose upon the whole mixture its own radiative equilibrium temperature. For the active gas will absorb radiation, and either pass it on to the neutral gas by a 'collision of the second sort' (Stoss zweiter Art) or re-emit it. Equilibrium between energy loss by impacts and gain from collisions will be established when the temperatures are equal. Hence we need only consider the 'active' gases water-vapour, carbon dioxide, ozone and oxygen.

Immediately above the troposphere, say from ro $\mathrm{km}$. to $50 \mathrm{~km}$., the effect of the oxygen will be unimportant. Its absorption in the visible region is small; the rays it can absorb in the far ultra-violet will have been filtered out by the oxygen above. The influence of ozone might be more important, but it will be far outweighed by the effect of the water-vapour and the carbonic acid, the concentration of which in this region is considerable. The concentration of water-vapour at Ir $\mathrm{km}$. will probably be about $2.5 \times 10^{-8} \mathrm{gm} . / \mathrm{cm} .{ }^{3}$, falling to about $4 \times 10^{-10} \mathrm{gm} . / \mathrm{cm}^{3}$ at $55 \mathrm{~km}$. The concentration of carbonic acid will be about $\mathrm{r} \cdot 2 \times 10^{-7} \mathrm{gm} . / \mathrm{cm} .^{3}$ at I I km., falling to about $7.5 \times$ I0 ${ }^{-12} \mathrm{gm}$. $/ \mathrm{cm}$. at $55 \mathrm{~km}$. The total amount of ozone above rr $\mathrm{km}$. amounts to about $x \cdot 4 \times 10^{-5}$ of the whole amount of oxygen above this level, but its exact distribution is at present unknown. Presumably it is formed by the sun's ultraviolet radiation, which is absorbed by oxygen at wavelengths below $2000 \AA$.U. In this case the amount formed per $\mathrm{cm} \cdot{ }^{3}$ must reach a maximum somewhere in the neighbourhood of $60 \mathrm{~km}$. At greater heights the decrease in the partial pressure of oxygen outweighs the increase in intensity of the solar radiation. At lesser heights so much of the initial radiation of the effective wavelength has been absorbed that its loss is not counterbalanced by the increased concentration of oxygen. Hence it is above $50 \mathrm{~km}$. that the concentration of ozone compared to the concentration of water-vapour and carbonic acid becomes important.

Now the equilibrium temperature of the air will be some sort of mean between the equilibrium temperatures in these three gases. The equilibrium temperature of carbon dioxide is easy to calculate. It has only one strong absorption band at $14 \cdot 6 \mu$, and its temperature will be found by equating the emission from this band to the absorption. Since there is very little energy in this part of the solar spectrum, the carbon dioxide only absorbs energy from the layers below, more especially the earth, whilst it radiates both upwards and downwards. Its equilibrium temperature is therefore low, about $236^{\circ}$. The value for water-vapour is not so easy to determine, on account of its complicated absorption spectrum. It also absorbs in the infra-red only, and 
therefore has a low equilibrium temperature, probably between $200^{\circ}$ and $220^{\circ}$. The case of ozone is even more complicated, since it absorbs not only at $9.5 \mu$ but also to a certain extent in the visible and very strongly in the ultra-violet below $3000 \AA$.U. Since a not inconsiderable fraction of the sun's radiation (probably more than I per cent.) lies in this region, this amount of heat as well as that abstracted from the visible part of the spectrum has to be accounted for in some form or other. It can only be re-emitted by pure ozone at a wave-length of $9.5 \mu$, and its equilibrium temperature when exposed to sunlight and the earth's radiation would therefore be correspondingly high, probably well above $300^{\circ}$.

The general picture may therefore be drawn in outline as follows. At low heights in the troposphere we have convection and therefore an adiabatic temperature gradient. Above Ix km., at which height the temperature has fallen to the equilibrium radiative temperature, i.e. $220^{\circ}$, convection will be damped out.
From here on one should have a compromise between the radiative equilibrium temperatures of the various active gases. At II km. the effect of water and carbon dioxide will preponderate and a low temperature will obtain, but as one ascends, the carbon dioxide disappears and the water diminishes, whilst the ozone with its high equilibrium temperature will become more and more dominant. Above $60 \mathrm{~km}$. the effect of ozones should outweigh that of the other gases and the air should approach the equilibrium temperature of this gas, i.e. about $300^{\circ}$, as is indicated by the various more empirical arguments we have outlined.

Whether the agreement of all these forms of indirect reasoning is regarded as convincing is of course a matter of personal opinion. Final certainty can only be attained by direct measurement. It is much to be desired that such immediate observations be made at the earliest possible moment, but the expense and difficulty at present seem to be prohibitive.

\section{Methods and Results of the American Museum Expeditions in the Gobi Desert, I922-25. ${ }^{1}$}

By Prof. Henry Fairfield Osborn, For. Mem. R.S. NEW volume in the life-history of the earth,
composed up to the present of twenty-four chapters (see table below), has been revealed by the discoveries of the Mongolian Expeditions of the American Museum of Natural History, under the leadership of Roy Chapman Andrews. Central Asia, and especially the region east and south-east of Chinese Turkestan, long remained the terra incognita of geology, palæontology, and, in a minor sense, of geography. In 1900 the lecturer predicted that the unknown highplateau region of Central Asia, rather than the wellknown Asiatic provinces on the south, such as the Siwalik Hills of India, explored by Hugh Falconer (1830-50), would prove to be the chief centre of the origin and distribution of the mammalia from which waves of north mammalian life radiated to the continents of Europe and of North America.

Andrews's expeditions in the three seasons of I922, 1923 , and 1925 , have not only completely verified this prediction, but have also revealed the high Central Asiatic plateau region as the chief home of the terrestrial deinosaurian reptiles of Upper Jurassic and of Cretaceous time. In brief, these discoveries establish Mongolia as a chief centre of northern terrestrial life-history, from the close of Jurassic time onwards to the very close of Pleistocene time.

From the point of view of palæogeography, the outstanding geological discoveries of the expedition are:

First, this Central Asiatic continent of Gobia, as it has been named by Grabau, was for several millions of years extremely favourable to the evolution of reptiles, mammals, insects, and plants, and probably birds as well, hitherto known along the low-lying Cretaceous forelands of western Europe (such as the Wealden of England and Belgium), and in less degree of southern Asia. Secondly, this now terribly desert region of Gobia, traversed only by the gazelle and the wild ass, and thoroughly uninhabitable in the summer season, was abounding in life throughout Upper

1 Abstract of a Jecture delivered by the author to the Geological Society of London on June 23

N O. 2962, VOL. I I 8]
Jurassic, and throughout all Cretaceous and Tertiary time, sparsely forested, traversed by streams and rivers, with a limited seasonal rain-supply like the highplateau region of Central Africa to-day. Thirdly, these dry and stimulating upland conditions of 'Tertiary time, as compared with the densely forested conditions of the Asiatic lowlands, have led to the recent prediction by the lecturer on returning from Iren Dabasu in I 923 , that this region is the most likely one in which to search for the Tertiary ancestors of man, namely, those of Eolithic or Dawn-stone Age, though no traces of man have, as yet, been discovered by the expedition older than those of Lower Palæolithic age. The discovery of human and pre-human remains in Tertiary time has thus become one of the chief remaining objects of the expedition.

During the season of 1925 a great culture-camp, probably of Azilian-Campignian time, was discovered on the eastern slopes of the Altai Range, not far from Shabarakh, and not far from Djadokhta, where the now famous deinosaur eggs were discovered, far north of the Ordos locality explored by Licent and Teilhard de Chardin. In fact, these Upper Palæolithic artisans collected the broken shells of the deinosaur eggs with which to manufacture necklace ornaments, these perforated fossil shells serving as well as the recent eggshells of the giant Struthiolithus, the great ostrich of the Stone Age of Mongolia.

No human fossils have so far been found: the industrial levels are not as yet precisely determinable, but the chief anthropological fact is established that the Stone Age tribes spread over the borders of the Gobi Desert region during the Ice Age, establishing their quarries near the large lakes bordering the Altai Mountains on the east and fed by glacial streams. The geologists of the party have discovered traces of this glacial age along the summits of the Altai Range.

As for methods, by combining a very large caravan for the camel transport, which leaves Kalgan on December I, and reaches the eastern base of the Altai Range on May I, with an automobile train of five to 